\title{
Buccal injection of synthetic HPV long peptide vaccine induces local and systemic antigen-specific CD8+ T-cell immune responses and antitumor effects without adjuvant
}

Ming-Chieh Yang ${ }^{1,2}$, Andrew Yang ${ }^{1}$, Jin Qiu ${ }^{1,3}$, Benjamin Yang ${ }^{1}$, Liangmei He ${ }^{1}$, Ya-Chea Tsai ${ }^{1}$, Jessica Jeang ${ }^{1}$, T.-C. Wu ${ }^{1,4,5,6}$ and Chien-Fu Hung ${ }^{1,6,7^{*}}$

\begin{abstract}
Background: Human Papillomavirus is responsible for over $99 \%$ of cervical cancers and is associated with cancers of the head and neck. The currently available prophylactic vaccines against HPV do not generate therapeutic effects against established HPV infections and associated lesions and disease. Thus, the need for a therapeutic vaccine capable of treating HPV-induced malignancies persists. Synthetic long peptides vaccination is a popular antigen delivery method because of its safety, stability, production feasibility, and its need to be processed by professional antigen presenting cells before it can be presented to cytotoxic CD8+ T lymphocytes. Cancers in the buccal mucosa have been shown to elicit cancer-related inflammations that are capable of recruiting inflammatory and immune cells to generate antitumor effects. In the current study, we evaluated the therapeutic potential of synthetic HPV long peptide vaccination in the absence of adjuvant in the TC-1 buccal tumor model.

Result: We show that intratumoral vaccination with E7 long peptide alone effectively controls buccal TC-1 tumors in mice. Furthermore, we observed an increase in systemic as well as local E7-specific CD8+ T cells in buccal tumorbearing mice following the vaccination. Finally, we show that induction of immune responses against buccal tumors by intratumoral E7 long peptide vaccination is independent of CD4+ T cells, and that the phenomenon may be related to the unique environment associated with mucosal tissues.
\end{abstract}

Conclusion: Our results suggest the possibility for clinical translation of the administration of adjuvant free therapeutic long peptide vaccine as a potentially effective and safe strategy for mucosal HPV-associated tumor treatment.

Keywords: Immunotherapy, E7 long peptide, Adjuvant free, Buccal tumor

\section{Background}

It is now clear that human papillomavirus (HPV) infection is responsible for over $99 \%$ of all cervical cancers, and is also associated with many other anogenital malignancies including vaginal and anal cancers [1]. In addition, the prevalence of HPV infection in head and neck cancers increased significantly within the past decade,

\footnotetext{
${ }^{*}$ Correspondence: chung2@jhmi.edu

${ }^{7}$ Departments of Pathology and Oncology, The Johns Hopkins University School of Medicine, CRB II Room 307, 1550 Orleans Street, Baltimore, MD 21231, USA

Full list of author information is available at the end of the article
}

with approximately $75 \%$ of diagnosed oropharyngeal cancers corresponding with HPV infection [2]. Among all HPV subtypes, the high-risk oncogenic HPV subtypes, predominantly HPV type 16 , are responsible for the majority of HPV associated cancer [3, 4]. The known etiology of HPV-associated diseases provides an excellent opportunity to develop vaccines against the highrisk HPV types. Encouragingly, there have been several successes in the development of prophylactic vaccines against disease-causing HPV subtypes [5]. However, these prophylactic vaccines can only prevent infections and do not generate therapeutic effects against 
established HPV infections and HPV-associated lesions [6]. Thus, the urgent need for the development of a therapeutic vaccine capable of treating HPV-induced malignancies persists.

To date, several clinical trials have been conducted using HPV-16 encoded oncoproteins E6 and E7 as targets of immunotherapy to treat HPV-induced cancers [7-10]. Among different therapeutic vaccine designs, peptide-based vaccines containing minimal epitopes of oncoproteins E6 and E7 have been popular and promising due to their safety, stability, and production feasibility $[9,11-13]$. However, some limitations to peptide vaccines dampen their application efficacy. Importantly, short peptides may be directly loaded onto any MHC I molecules on the surface of cells, including those that are not professional antigen presenting cells (APCs). This may result in interaction between $\mathrm{T}$-cell receptor and MHC I-antigen peptide complex in the absence of costimulation, causing T-cell anergy and immune tolerance [13]. To overcome this issue and increase the efficacy of the peptide vaccine, the length of the peptide antigen has been increased $[14,15]$. The synthetic long peptides are too large for the direct loading onto MHC I molecules on the surface of cells, thus requiring the peptide to be taken up and processed before the epitope can be presented on MHC I molecules, which is a process unique to the professional APCs. The professional APCs, such as the dendritic cells (DCs), can also provide the co-stimulatory signals during antigen presentation, ensuring quality $\mathrm{T}$ cell activation $[16,17]$.

Despite the improved antigen presentation process of the synthetic long peptide vaccine, the issue of poor immunogenicity remains. Typically, additional adjuvant or immunostimulant is required to induce the desired immune responses for vaccines incorporating synthetic peptides of a target antigen [9]. It is well known that malignant tumors, including squamous cell carcinomas of head and neck, are strongly associated with local inflammation [18, 19]. These cancer-related inflammations trigger the release of cytokines and the recruitment of inflammatory and immune cells, which could lead to the induction of either immune suppression or antitumor immunity $[20,21]$. Thus, the inflammatory nature of cancer may potentially serves as self-adjuvant capable of inducing the antigen-specific immune responses following synthetic long peptide vaccination.

In the current study, we evaluated the therapeutic potential of a synthetic HPV long peptide vaccine in the absence of adjuvant in the TC-1 buccal tumor model. We showed that intratumoral (I.T.) vaccination with HPV-16 E7aa 43-62 synthetic long peptide lead to enhanced antitumor effect in buccal tumor-bearing mice in the absence of adjuvant administration. Furthermore, we observed an increase in the number of E7-specific CD8+ T cells in the peripheral blood, spleen, and the buccal mucosa tissue. We also observed that the antitumor effect of the synthetic long peptide vaccination is CD8+ T cells dependent and $\mathrm{CD} 4+\mathrm{T}$ cells independent. We also showed that in comparison to subcutaneous tumor model, intratumoral synthetic long peptide vaccination in the absence of adjuvant lead to the generation of superior E7-specific CD8+ T cell response as well as more potent therapeutic antitumor effects against tumors located in the buccal mucosal region. Finally, we demonstrated that the observed therapeutic effects generated by intratumoral E7 long peptide vaccination in the buccal area are abolished upon deletion of toll-like receptor 4. Our results indicate that adjuvant free therapeutic long peptide vaccination is an effective and safe therapeutic strategy for treating tumors located in the mucosa.

\section{Results}

Intratumoral administration of synthetic HPV long peptide vaccine in the buccal area generates potent antitumor responses

First, we assessed the antitumor effect generated by intratumoral synthetic HPV-16 E7aa 43-62 peptide vaccination in the HPV-16 E7-expressing TC-1 buccal tumor model. C57BL/6 mice (five per group) were challenged with $3 \times 10^{4} \mathrm{TC}$-1-Luc cells in the right buccal area, then vaccinated I.T. with or without synthetic HPV-16 E7aa 43-62 peptide 3 days after for 4 times with a 4 day interval (Fig. 1a). As shown in Fig. 1b, mice vaccinated with E7 peptide generated significantly better antitumor effects which resulted in tumor control as measured by bioluminescence intensity compared to untreated mice. In addition, the vaccinated mice demonstrated longer survival compared to untreated mice (Fig. 1c). However, when the tumor bearing mice were vaccinated with non-specific CTL peptide (OVA 241-270), neither effective tumor control (Additional file 1: Figure S1A-B) nor prolonged survival (Additional file 1: Figure S1C) were observed. These data indicated that intratumoral synthetic E7 long peptide vaccination leads to effective E7-expressing buccal tumor control in the absence of additional adjuvant.

\section{Intratumoral administration of synthetic HPV long peptide vaccine in the buccal area leads to generation of systemic and local E7-specific CD8+ T cell responses}

We next evaluate the potential of I.T. E7aa 43-62 long peptide vaccination in generating antigen-specific adaptive immune responses. C57BL/6 mice (five per group) were challenged with $3 \times 10^{4} \mathrm{TC}$-1-Luc cells in the right buccal area, then vaccinated I.T. with or without synthetic E7aa 43-62 peptide 3 days after for four times with a 4 day interval. Peripheral blood, spleen, and tumors 

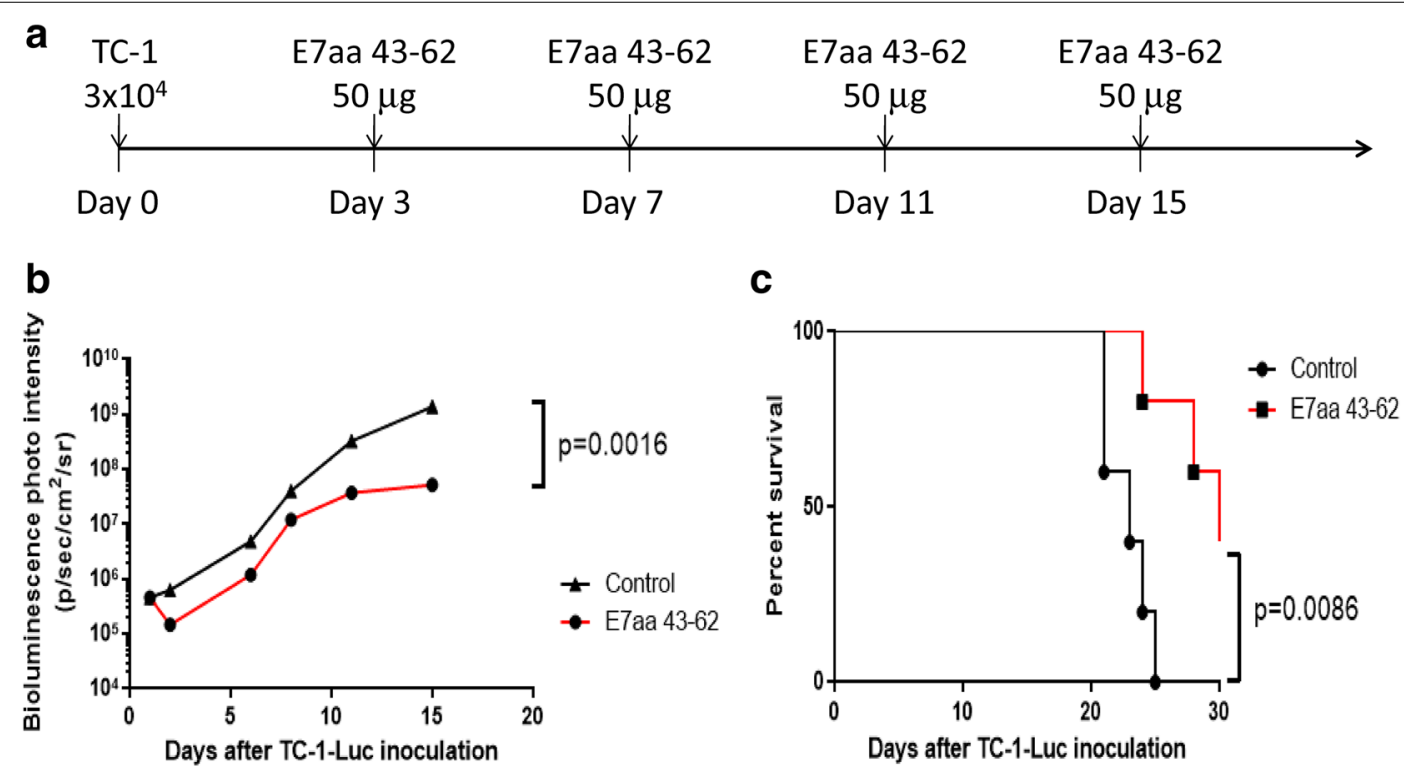

Fig. 1 Characterization of antitumor effect in tumor bearing mice treated with intratumoral synthetic HPV-16 E7aa 43-62 long peptide vaccination in buccal mucosal region. $3 \times 10^{4} \mathrm{TC}-1$-Luc cells were submucosally injected into the right buccal area of C57BL/6 mice (five per group). Three days after tumor injection, mice were vaccinated intratumorally with or without $50 \mathrm{\mu g}$ of synthetic HPV-16 E7aa 43-62 peptide for four times in a 4-day intervals. a Schematic diagram of treatment regimens. $\mathbf{b}$ Line graph depicting the change in mean luminescence intensity of tumor bearing mice after tumor injection (mean \pm SD). c Kaplan-Meier survival analysis of mice

of the tumor bearing mice were harvested 21 days after tumor challenge and either stained with E7 tetramer or stimulated with E7 peptide followed by IFN- $\gamma$ staining and analyzed by flow cytometry. As shown in Fig. 2a, $\mathrm{b}$, more E7-specific CD8+ T cells were induced in the peripheral blood of tumor bearing mice vaccinated with E7aa 43-62 long peptide compare to mice without vaccination. Mice vaccinated with E7aa 43-62 long peptide also generated more IFN- $\gamma$ secreting CD8 $+\mathrm{T}$ cells in spleen compared to untreated mice (Fig. 2c). Furthermore, as shown in Fig. 2d, mice vaccinated with E7aa 43-62 long peptide also generated more E7-specific $\mathrm{CD} 8+$ in the buccal tumor compared to untreated mice. Taken together, these data suggest that intratumoral synthetic long peptide vaccination can induce potent systemic E7-specific CD8 + T cell generation as well as local antigen-specific CD8 $+\mathrm{T}$ cells accumulation in buccal tumor in the absence of adjuvant.

The synthetic HPV long peptide vaccine generates potent antitumor effects against HPV-16 E7 expressing tumors in CD4-depleted mice but not in CD8-depleted mice Next, we sought to determine which immune cell population is predominately responsible for the generation of antitumor effects following synthetic E7 long peptide vaccination. We repeated the experiment in both CD4 depleted mice and CD8 depleted mice. As shown in Fig. 3b, c, CD8 depleted mice showed significantly increased tumor growth as measured by bioluminescence intensity as well as reduction in survival even after synthetic long peptide vaccination compared to CD8 intact mice, at a level similar to the untreated mice. However, the treatment effects of tumor reduction and prolonged survival were observed in CD4 depleted, E7 long peptide vaccinated mice compared to mice without CD4 depletion (Fig. 3d, e). These results indicate that the antitumor responses elicited by I.T. vaccination of E7 long peptide in the absence of adjuvants are CD8 $+\mathrm{T}$ cells dependent and CD4+ T cells independent.

Intratumoral administration of synthetic HPV long peptide vaccine leads to better generation of E7-specific CD8+ $T$ cells and more potent antitumor effects against buccal mucosal tumor compared to subcutaneous tumor

Finally, we tested whether I.T. synthetic E7 long peptide vaccination without adjuvant can also elicit an antitumor immune response in other tumor models. C57BL/6 mice (five per group) were challenged with TC-1-Luc cells either submucosally in the right buccal area or subcutaneously in the abdomen and then vaccinated I.T. with synthetic E7 long peptide. 21 days after tumor challenges, the spleen of mice were harvested and stimulated with E7 peptide followed by IFN- $\gamma$ staining and analyzed by flow cytometry. As shown in Fig. 4a, b, I.T. synthetic 
a
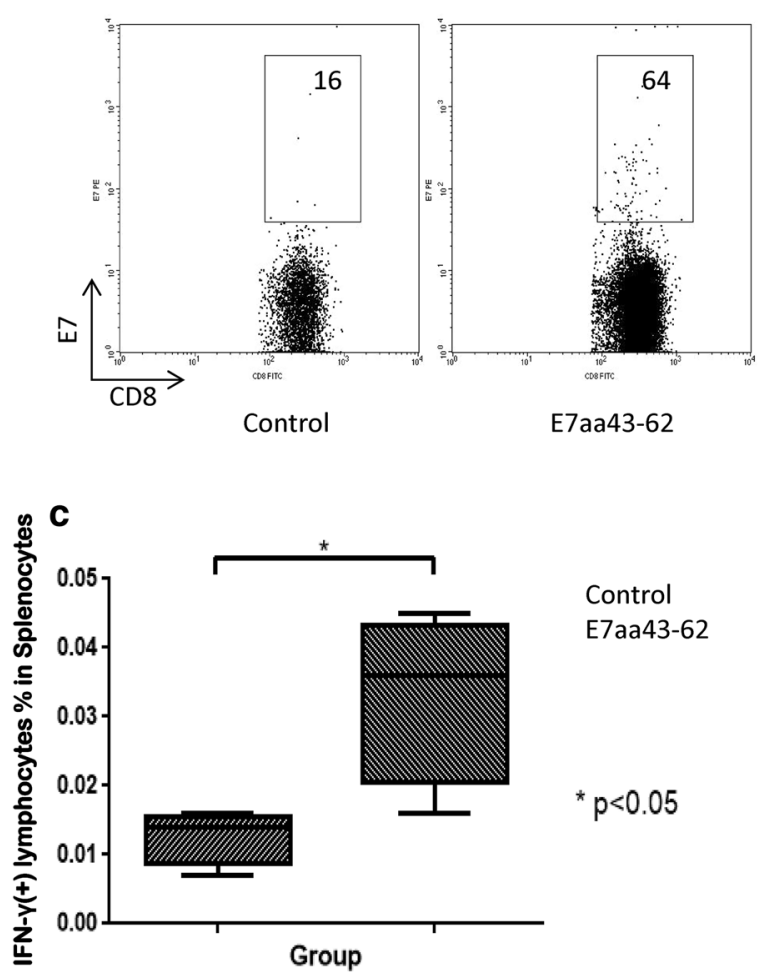

b

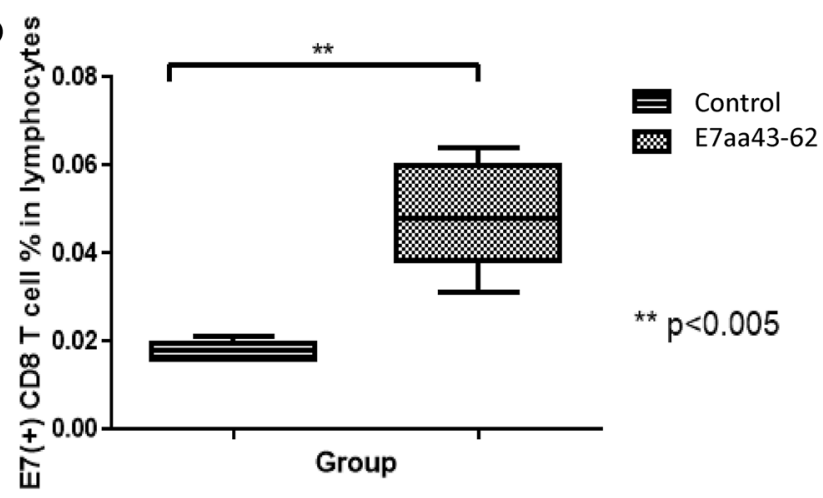

d

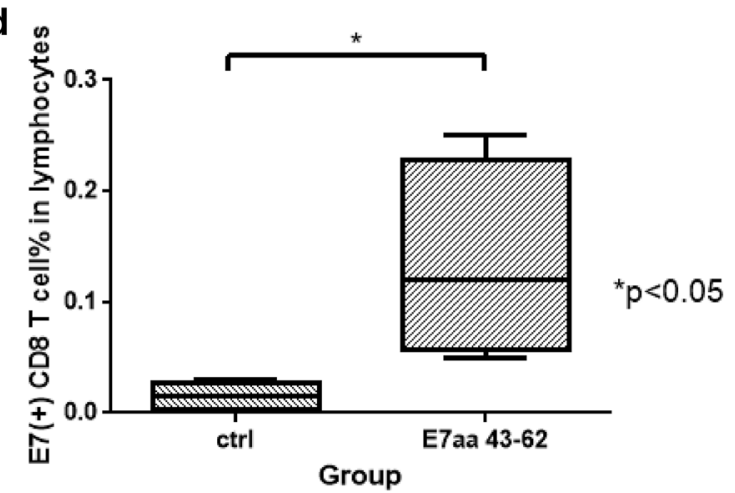

Fig. 2 Characterization of systemic E7-specific CD8+T cells and local E7-specific activated CD8+T cells in tumor bearing mice. $3 \times 10^{4} \mathrm{TC}-1$-LuC cells were submucosally injected into the right buccal area of C57BL/6 mice (five per group). Three days after tumor injection, mice were vaccinated intratumorally with or without $50 \mu \mathrm{g}$ of synthetic HPV-16 E7aa 43-62 peptide for four times in a 4-day intervals. 21 days after tumor injection, peripheral blood was collected and the spleen and tumor were harvested. Cells obtained from the peripheral blood and tumor were stained with PE-conjugated HPV16 H-2D-RAHYNIVTF tetramer and APC-conjugated CD8 monoclonal antibody followed by flow cytometry analysis. Spleenocytes were stimulated with HPV16 E7aa49-57 peptide in the presence of GolgiPlug and IFN- $\gamma$-secreting CD8+ T cells were detected by intracellular cytokine staining followed by a flow cytometry analysis. a Representative flow cytometry images showing the amount of E7-specific CD8+ T cells per $1 \times 10^{5}$ lymphocytes in the peripheral blood of various groups. b Bar graph depicting the amount of E7-specific CD8+ T cells per $1 \times 10^{5}$ lymphocytes in the peripheral blood of various groups (mean \pm SD). $\mathbf{c}$ Bar graph depicting the amount of IFN- $y$ positive lymphocytes per $1 \times 10^{5}$ splenocytes (mean \pm SD). d Bar graph depicting the percentage of E7-specific CD8+ T cells in all lymphocytes in the buccal tumor (mean \pm SD)

long peptide vaccination in buccal tumor-bearing mice generated significantly higher amount of IFN- $\gamma$ secreting $\mathrm{CD} 8+\mathrm{T}$ cells in the spleen compared to naïve mice, mice received buccal vaccination alone or buccal tumor challenge alone, whereas there were no significant differences in the number of IFN- $\gamma$ secreting CD8 + T cells in spleen of mice receiving subcutaneous treatments. Furthermore, mice challenged with buccal tumor injection and treated with I.T. synthetic E7 long peptide vaccination showed significantly reduced tumor growth compared to mice without treatment, whereas I.T. synthetic E7 long peptide vaccination was not able to control tumor growth in mice challenged with subcutaneous tumor injection (Fig. 5a, b). These data demonstrated that I.T. synthetic E7 long peptide vaccination without adjuvant supplementation is more effective in the treatment of tumors located in the buccal mucosa than in the treatment of tumors located in the subcutaneous abdomen.
Knocking out toll-like receptor 4 abolishes the therapeutic effect of intratumoral synthetic HPV long peptide vaccination

As mentioned in the introduction, the inflammatory nature of tumors located in the buccal area may serve as an adjuvant to induce antitumor immunity. Thus, the innate immune environment of the buccal area may play a role in mediating the generation of antigenspecific CD8+ $T$ cell responses and anti-tumor effects observed from previous experiments involving intratumoral synthetic E7 long peptide vaccination against buccal TC-1 tumors. To examine the importance of innate immune responses in the observed treatment effect, we repeated the same experiment described in Fig. 1 in C57BL/10ScNJ (TLR4 deficient) mice. As shown in Fig. 6, TLR4 -/ - mice treated with intratumoral synthetic E7 long peptide vaccination did not generate significant antitumor effect against buccal TC-1 tumor to 


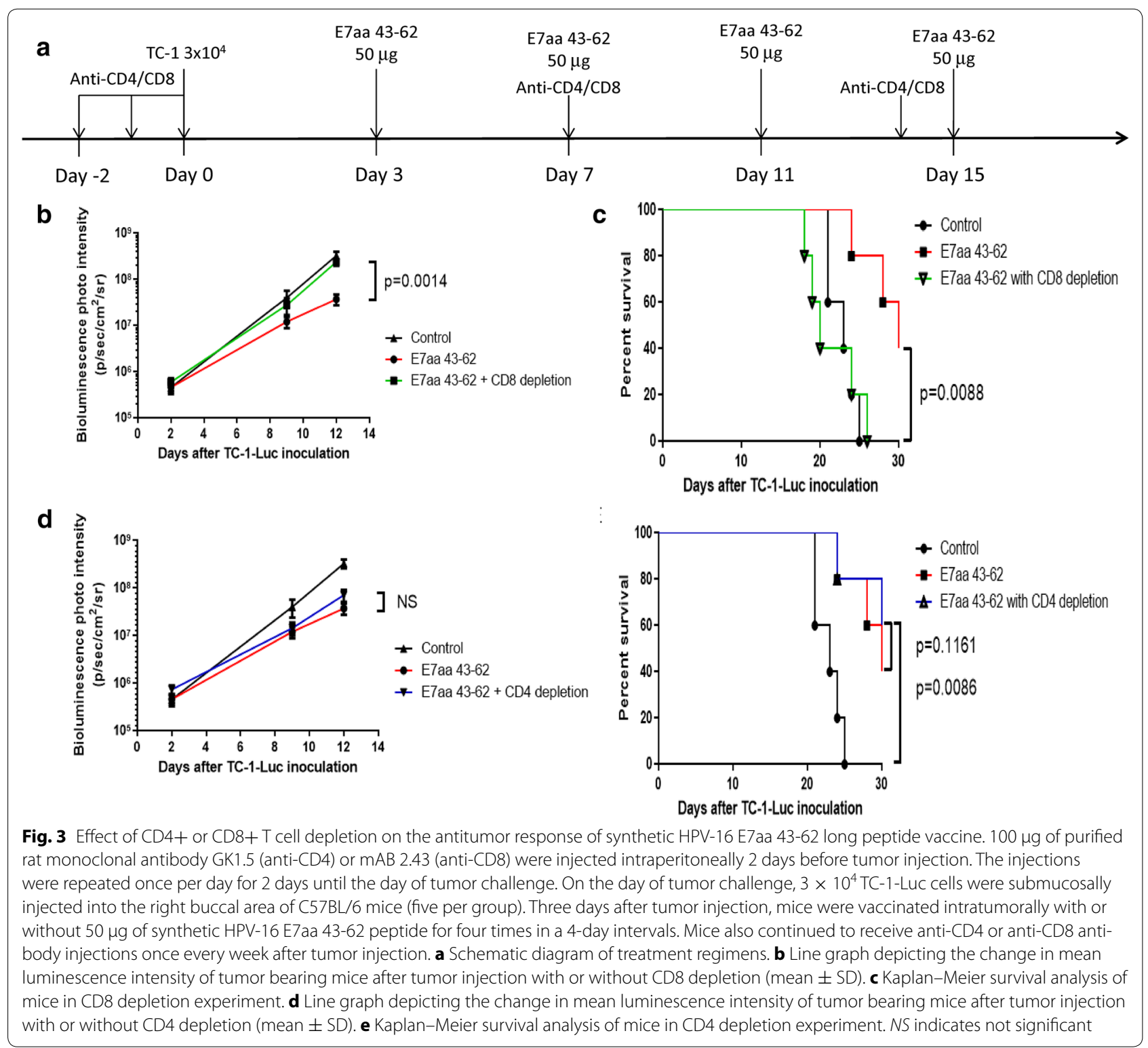

prolong survival compared to the control, untreated mice. Buccal tumor-bearing mice with intact TLR4 function and received intratumoror synthetic E7 long peptide vaccination, however, survived significantly longer than TLR4 deficient mice who received the same treatment. This observation supports the hypothesis that the innate immune system of the buccal area is important for the immune responses and anti-tumor effects elicited by intratumoral E7 long peptide vaccination against tumors located in the buccal mucosa area.

\section{Discussion}

In the current study, we examined the effects of adjuvant free, I.T. synthetic E7 long peptide vaccination on the generation of antigen-specific immune responses and antitumor effects. We observed that following I.T. synthetic E7 long peptide vaccination, the buccal tumorbearing mice exhibited significant increase in systemic and local E7-specific CD8+ T cells, effectively controlling the tumor growth. In addition, we found that the antitumor effects generated by E7 long peptide vaccine is predominantly mediated by the CD8+ T cells and not by CD4+ T cells. Finally, we show that I.T. synthetic E7 long peptide vaccination without adjuvant elicited a better E7-specific CD8+ T cell response and more potent antitumor effects against tumors located in the buccal mucosa than to tumors located in the subcutaneous abdomen. 

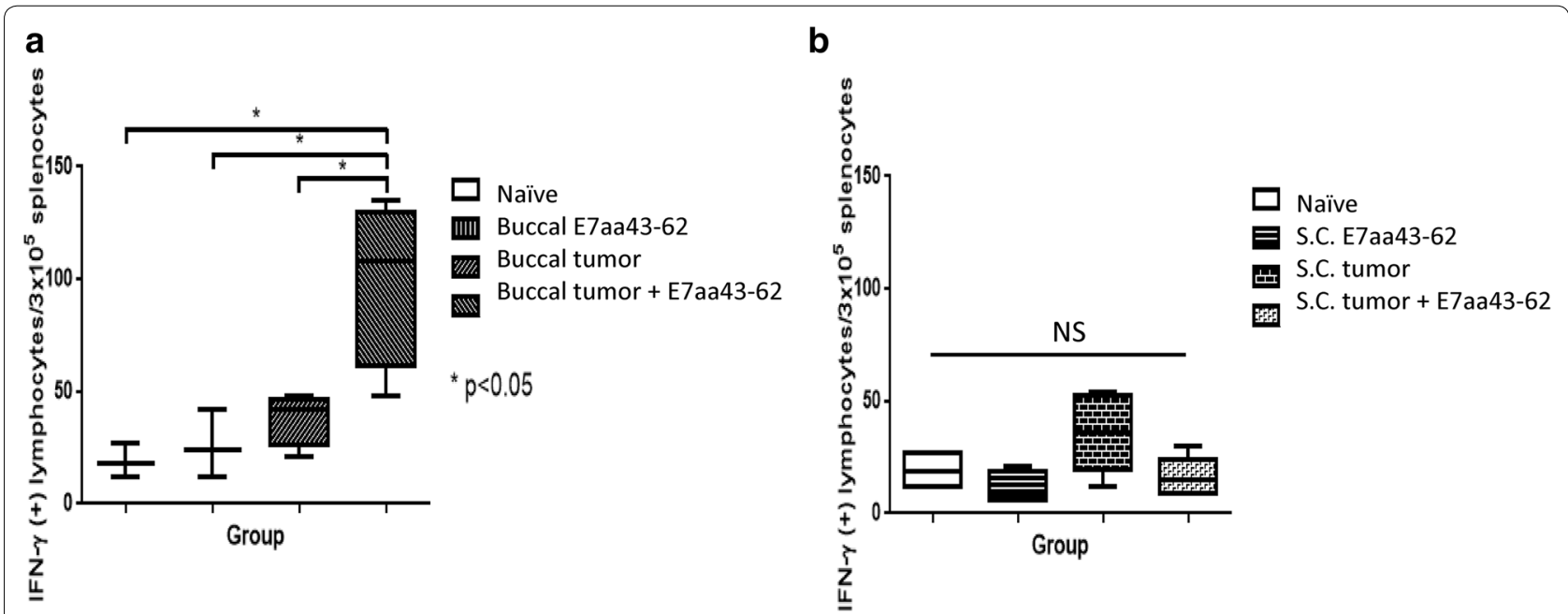

Fig. 4 Comparison of HPV-16 E7 specific CD8+ T cell responses induced by synthetic HPV-16 E7aa 43-62 long peptide vaccine in various tumor model. C57BL/6 mice (five per group) received either $3 \times 10^{4} \mathrm{TC}-1$-Luc cells injection submucosally into the right buccal area or $1 \times 10^{5} \mathrm{TC}-1$-LuC cells injection subcutaneously into the abdomen. Three days after tumor injection, mice were vaccinated intratumorally with or without $50 \mu \mathrm{g}$ of synthetic HPV-16 E7aa 43-62 peptide for four times in a 4-day intervals. 21 days after tumor injection, spleenocytes were harvested and stimulated with HPV16 E7aa49-57 peptide in the presence of GolgiPlug and IFN- $\gamma$-secreting CD8+ T cells were detected by intracellular cytokine staining followed by a flow cytometry analysis. a Bar graph depicting the amount of IFN- $\gamma$ positive lymphocytes per $3 \times 10^{5}$ splenocytes for buccal treatments (mean \pm SD). b Bar graph depicting the amount of IFN- $\gamma$ positive lymphocytes per $3 \times 10^{5}$ splenocytes for subcutaneous treatments (mean \pm SD). NS indicates not significant

Of note, we demonstrated a difference in the ability of E7 long peptide vaccine in generating potent antigenspecific immune responses and antitumor effects when vaccinated I.T. against buccal tumors compared to when vaccinated I.T. against subcutaneous tumors (Figs. 4 and 5). This observation is supported by previous data. We have previously explored the employment of a sulfated polysaccharide compound from red algae, carrageenan, as adjuvant to generate antigen-specific immune responses and antitumor effects following subcutaneous E7 peptide vaccination [22]. The experiment demonstrated that subcutaneous E7 peptide vaccination alone without carrageenan administration did not lead to generation and activation of E7-specific CD8+ T cell immune response, which correspond to limited protective and therapeutic antitumor effects. In a separate study, we explored the adjuvant effect of chemotherapy in eliciting antigenspecific antitumor response and showed that I.T. vaccination of E7 peptide without cisplatin administration did not lead to effective control of subcutaneous tumors or the generation of potent E7-specific immune responses [23]. We explored the potential reasons for the phenomena observed in these previous studies, and showed that there was significantly less CD11c+ DCs accumulation in tumor as well as DCs migration to the lymph nodes when vaccinated I.T. with E7 peptide only without administration of adjuvant $[22,23]$. Furthermore, DCs isolated from mice treated with E7 peptide vaccine alone express significantly less costimulatory molecules compared to those isolated from mice treated with both peptide vaccination and adjuvant administration, which translates into a lower ability to activate E7-specific CD8+ T cells. In contrast, a previous study has shown that buccal immunization with measles virus nucleoprotein (NP) alone is capable of eliciting a NP-specific CD8+ CTL response [24]. Furthermore, the study observed a rapid recruitment of DCs into the buccal mucosa after NP vaccination. In this study, we showed that the innate immune system regulated by TLR4 plays a significant role in eliciting the antitumor responses against buccal TC-1 tumor (Fig. 6). The differences in the tissue environment and the ability to recruit APCs to the local area may account for the difference in the generation of immune responses and antitumor effects between I.T. vaccination against buccal tumor versus I.T. vaccination against subcutaneous tumor. The trafficking of immune cells to the tumor location before and after I.T. E7 peptide vaccination in the buccal mucosa or subcutaneous abdomen should be further explored in future studies.

One key finding of the current study is that the experiments involving synthetic E7 long peptide vaccination were conducted without administration of supplementing adjuvants. Many studies have been performed to explore different approaches to elicit potent immune responses and antitumor effects through adjuvant-free vaccination [25-27]. Even though administration of 


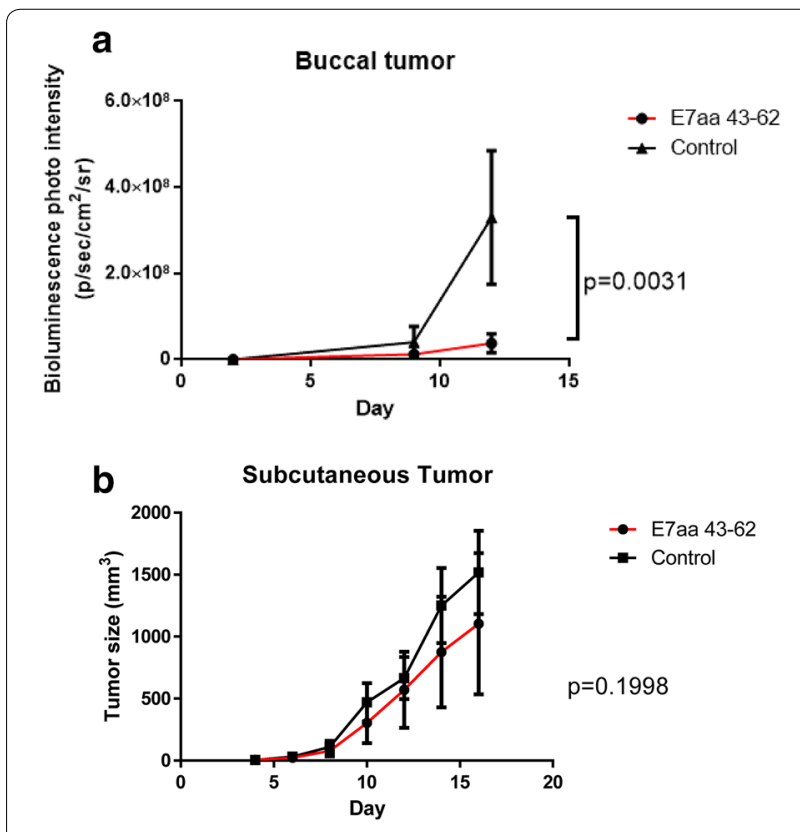

Fig. 5 Comparison of antitumor effects induced by synthetic HPV-16 E7aa 43-62 long peptide vaccine in various tumor model. C57BL/6 mice (five per group) received either $3 \times 10^{4} \mathrm{TC}-1$-Luc cells injection submucosally into the right buccal area or $1 \times 10^{5} \mathrm{TC}-1$-Luc cells injection subcutaneously into the abdomen. Three days after tumor injection, mice were vaccinated intratumorally with or without $50 \mu \mathrm{g}$ of synthetic HPV-16 E7aa 43-62 peptide for four times in a 4-day intervals. a Line graph depicting the change in mean luminescence intensity of tumor bearing mice after buccal tumor injection (mean $\pm \mathrm{SD}$ ). $\mathbf{b}$ Line graph depicting the change in mean luminescence intensity of tumor bearing mice after subcutaneous tumor injection (mean $\pm \mathrm{SD}$ )

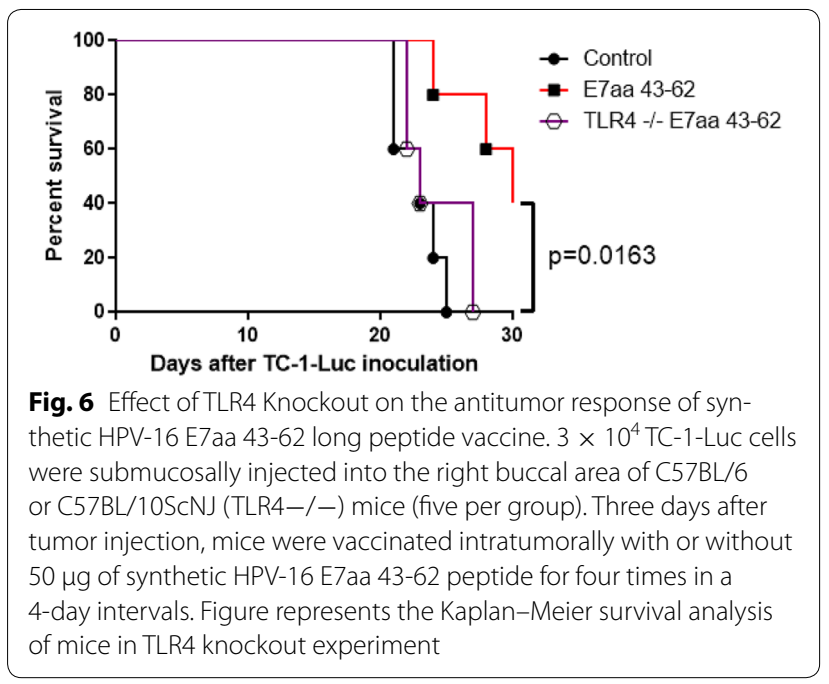

adjuvants can elicit stronger immune responses, many substances with adjuvant effects have also been shown to have negative impact on tumor treatment $[28,29]$, to cause $\mathrm{T}$ cells dysfunction and retention [30, 31], to have neurotoxicity [32], or to induce autoimmunity [33]. Thus, identifying appropriate adjuvants that are both safe and effective when incorporated into vaccination strategies is a significant concern. Our study suggests the potential utilization of the natural immunogenic characteristics of mucosal tissue to elicit potent antigen-specific immune responses as well as therapeutic antitumor effects without administration of adjuvants, thus reducing the safety concerns for vaccination.

\section{Conclusion}

In summary, we found that intratumoral therapeutic synthetic E7 long peptide vaccination resulted in both systemic and local increase of antigen-specific CD8+ $\mathrm{T}$ cells in mice bearing buccal tumors without the need to administer additional adjuvants to enhance the immunogenicity of the vaccine. This study suggests the possibility of clinical translation of administration of an adjuvant-free therapeutic HPV vaccine to generate potent cell-mediated immune responses and antitumor effects against HPV-associated lesions while preventing potential complications caused by adjuvants.

\section{Methods}

\section{Mice}

Six- to eight-week-old female C57BL/6 mice were purchased from the Charles Rivers Laboratories (Frederick, MD, USA). Female C57BL/10ScNJ mice carrying a spontaneous deletion of Tlr4 gene were obtained from The Jackson Laboratory (Bar Harbor, Maine, USA). All animal procedures were performed according to approved protocols at the Johns Hopkins Institutional Animal Care and Use Committee in accordance with recommendations for the proper use and care of laboratory animals.

\section{Cells}

TC-1 cells expressing luciferase (TC-1-Luc) were generated and cultured using methods described previously. $[34,35]$

\section{Synthetic long peptide vaccine}

The synthetic long-peptide vaccine used in this study, E7aa 43-62, consists of synthetic peptide resembling 
43-62 amino acid peptide chain of HPV-16 E7 antigen. This synthetic peptide construct contains an $\mathrm{H}-2 \mathrm{D}^{\mathrm{b}}$ restricted E7 epitope (aa 49-57), and its immunogenicity has been demonstrated in our previous study [36]. The synthetic peptide was prepared at $95 \%$ purity. No additional adjuvant was included in the vaccine.

For non-specific peptide vaccination experiment, CTL peptide OVA 241-270 (SMLVLLPDEVSGLEQLESIINF EKLTEWTS(OVA30)) were used. This peptide has been previously described [14].

\section{In vivo tumor treatment experiments}

For the establishment and treatment of orophoryngeal tumor, $3 \times 10^{4} \mathrm{TC}$-1-Luc cells were submucosally injected into the right buccal area of $\mathrm{C} 57 \mathrm{BL} / 6$ or $\mathrm{C} 57 \mathrm{BL} / 10 \mathrm{ScNJ}$ mice. Tumor growth was confirmed by IVIS2000 bioluminescence imaging system. Three days after tumor injection, mice were vaccinated intratumorally with $50 \mu \mathrm{g}$ of synthetic HPV-16 E7aa 43-62 peptide or $50 \mu \mathrm{g}$ of CTL peptide OVA 241-270. Mice received same booster vaccination at 4-day intervals for a total of three boosters. The luminescence intensity of tumor was measured routinely with IVIS imaging machine.

For the establishment and treatment of abdominal tumor, $1 \times 10^{5} \mathrm{TC}-1$-Luc cells were subcutaneously injected into the abdomen of C57BL/6 mice. Mice then received intratumoral vaccination using the same treatment schedule as described for the orophoryngeal tumor model. Tumor growth was monitored routinely by palpation and inspection.

\section{CD4/CD8 depletion}

Hundred microgram of purified rat monoclonal antibody GK1.5 (anti-CD4) or mAB 2.43 (anti-CD8) were injected intraperitoneally 2 days before tumor injection. The injections were repeated once per day for 2 days until the day of tumor challenge. Mice were then inoculated with TC-1-Luc cells and vaccinated with synthetic HPV-16 E7aa 43-62 peptide following the same treatment schedule as described earlier for the oropharyngeal tumor model. Mice continued to receive anti-CD4 or anti-CD8 antibody injections once every week after tumor injection.

\section{Peripheral blood cell, splenocyte, and tumor infiltrating lymphocyte preparation}

Twenty-one days after tumor injection, peripheral blood was obtained from the mice treated with various treatment regimen and the spleen and TC- 1 tumors of the mice were harvested. For the preparation of splenocytes, the spleen was meshed through a $70 \mu \mathrm{m}$ nylon filter mesh. The splenocytes and peripheral blood cells were treated with ACK lysis buffer to lyse the red blood cells, the cells were then washed and viable cells were identified using trypan blue dye exclusion. TC-1 tumors were surgically excised using sterile technique, placed in RPMI-1640 medium containing $100 \mathrm{U} / \mathrm{ml}$ penicillin and $100 \mu \mathrm{g} / \mathrm{ml}$ streptomycin and washed with PBS. The solid tumors were then minced into 1- to 2-mm pieces and immersed in serum-free RPMI-1640 medium containing $0.05 \mathrm{mg} / \mathrm{ml}$ collagenase I, $0.05 \mathrm{mg} / \mathrm{ml}$ collagenase IV, $0.025 \mathrm{mg} / \mathrm{ml}$ hyaluronidase IV, $0.25 \mathrm{mg} / \mathrm{ml}$ DNase I, $100 \mathrm{U} / \mathrm{ml}$ penicillin, and $100 \mu \mathrm{g} / \mathrm{ml}$ streptomycin and incubated at $37{ }^{\circ} \mathrm{C}$ with periodic agitation. The tumor digest was then filtered through a $70 \mu \mathrm{m}$ nylon filter mesh to remove undigested tissue fragments. The resultant single tumor cell suspensions and tumorinfiltrating lymphocytes were washed twice in Hank's buffered salt solution (HBSS) (400 g for $10 \mathrm{~min}$ ), and viable cells were determined using trypan blue dye exclusion.

\section{Tetramer analysis of E7-specific CD8+ T cells in tumor bearing mice}

Cells harvested from the peripheral blood and TC-1 tumors were stained with phycoerythrin (PE)-conjugated HPV16 H-2D-RAHYNIVTF tetramer combined with surface staining using APC-Alexa Fluor-conjugated anti-CD8 (BD Pharmingen). Cells were analyzed on a BD FACSCalibur collecting 500,000 events.

\section{Intracellular cytokine staining and flow cytometry analysis}

The cells harvested from the spleen of mice treated with various treatment regiments were incubated $0.1 \mu \mathrm{g} / \mathrm{ml}$ of HPV-16 E7 peptide containing an MHC class I epitope (aa49-57, RAHYNIVTF) in the presence of GolgiPlug (BD Pharmingen, San Diego, California, USA). The stimulated cells were then washed once with FACScan buffer and stained with phycoerythrin-conjugated monoclonal rat anti-mouse CD8a (clone 53.6.7). Cells were subjected to intracellular cytokine staining using the Cytofix/Cytoperm kit according to the manufacture's instruction (BD Pharmingen). Intracellular IFN- $\gamma$ was stained with FITCconjugated rat anti-mouse IFN- $\gamma$. All antibodies were purchased from BD Pharmingen. Flow cytometry analysis was done using FACSCalibur with CellQuest software (BD Bioscience). FITC Rat IgG1, к Isotype Control (Clone R3-34) was purchased from BD Pharmingen (Cat.\# 554684).

\section{Statistical analysis}

All data presented in this study are expressed as mean \pm SD. At least three samples per group were included in each of these experiments. Flow cytometry data and results of tumor treatment experiments were evaluated by analysis of variance (1-way ANOVA) and the Tukey-Kramer test. Individual data points were compared by student's $t$-test. For all analysis, $p$ values $<0.05$ were considered significant. 


\section{Additional file}

Additional file 1: Figure S1. Characterization of antitumor effect in tumor bearing mice treated with intratumoral non-specific OVA long peptide vaccination in buccal mucosal region. $3 \times 10^{4} \mathrm{TC}-1$-Luc cells were submucosally injected into the right buccal area of C57BL/6 mice (five per group). Three days after tumor injection, mice were vaccinated intratumorally with or without $50 \mu \mathrm{g}$ of CTL peptide OVA241-270 for four times in a 4-day intervals. (A) Luminescence images of mice challenged with TC-1-Luc tumor and treated with or without OVA peptide vaccinations. (B) Line graph depicting the change in mean luminescence intensity of tumor bearing mice after tumor injection (mean \pm SD). (C) Kaplan-Meier survival analysis of mice.

\section{Abbreviations}

HPV: human papillomavirus; SLPs: synthetic long peptides; APCs: antigen presenting cells; CTL: cytotoxic T lymphocyte; I.T: intratumoral; MHC: major histocompatibility complex; DCs: dendritic cells; IFN: interferon.

\section{Authors' contributions}

MCY, TCW, and CFH conceived and designed experiments and interpreted data. MCY, AY, JQ, BY, LH and YCT performed and experiments. MCY, AY, BY, JJ, $\mathrm{TCW}$, and CFH wrote the manuscript. All authors read and approved the final manuscript.

\section{Author details}

${ }^{1}$ Department of Pathology, Johns Hopkins Medical Institutions, Baltimore, MD, USA. ${ }^{2}$ Department of Surgery, Kaohsiung Veterans General Hospital, Kaohsiung, Taiwan. ${ }^{3}$ Department of Obstetrics and Gynecology, Shanghai Tenth People's Hospital of Tongji University, Shanghai, China. ${ }^{4}$ Department of Obstetrics and Gynecology, Johns Hopkins Medical Institutions, Baltimore, MD, USA. ${ }^{5}$ Department of Molecular Microbiology and Immunology, Johns Hopkins Medical Institutions, Baltimore, MD, USA. ${ }^{6}$ Department of Oncology, Johns Hopkins Medical Institutions, Baltimore, MD, USA. ${ }^{7}$ Departments of Pathology and Oncology, The Johns Hopkins University School of Medicine, CRB II Room 307, 1550 Orleans Street, Baltimore, MD 21231, USA.

\section{Acknowledgements}

This work was supported by the United States National Institutes of Health (NIH) Cervical Cancer Specialized Program of Research Excellence (SPORE) (P50 CA098252), R01 grant (CA114425-01), R21 grant (CA194896-01), and Al109259-01 grant.

\section{Competing interests}

The authors declare that they have no competing interests.

Received: 9 October 2015 Accepted: 15 February 2016 Published online: 03 March 2016

\section{References}

1. Bosch FX, Lorincz A, Munoz N, Meijer CJ, Shah KV. The causal relation between human papillomavirus and cervical cancer. J Clin Pathol. 2002;55:244-65.

2. Mehanna H, Beech T, Nicholson T, El-Hariry I, McConkey C, Paleri V, Roberts S. Prevalence of human papillomavirus in oropharyngeal and nonoropharyngeal head and neck cancer-systematic review and metaanalysis of trends by time and region. Head Neck. 2013;35:747-55.

3. Munoz N, Bosch FX, de Sanjose S, Herrero R, Castellsague X, Shah KV, Snijders PJ, Meijer CJ. Epidemiologic classification of human papillomavirus types associated with cervical cancer. N Engl J Med. 2003;348:518-27.

4. Garbuglia AR. Human papillomavirus in head and neck cancer. Cancers (Basel). 2014;6:1705-26.

5. Wheeler CM. Advances in primary and secondary interventions for cervical cancer: human papillomavirus prophylactic vaccines and testing. Nat Clin Pract Oncol. 2007:4:224-35.
6. Hildesheim A, Herrero R, Wacholder S, Rodriguez AC, Solomon D, Bratti MC, Schiller JT, Gonzalez P, Dubin G, Porras C, et al. Effect of human papillomavirus 16/18 L1 viruslike particle vaccine among young women with preexisting infection: a randomized trial. JAMA. 2007;298:743-53.

7. Tran NP, Hung CF, Roden R, Wu TC. Control of HPV infection and related cancer through vaccination. Recent Results Cancer Res. 2014;193:149-71.

8. Ma B, Maraj B, Tran NP, Knoff J, Chen A, Alvarez RD, Hung CF, Wu TC. Emerging human papillomavirus vaccines. Expert Opin Emerg Drugs. 2012;17:469-92.

9. Liu TY, Hussein WM, Toth I, Skwarczynski M. Advances in peptide-based human papillomavirus therapeutic vaccines. Curr Top Med Chem. 2012;12:1581-92.

10. Bolhassani A, Mohit E, Rafati S. Different spectra of therapeutic vaccine development against HPV infections. Hum Vaccin. 2009;5:671-89.

11. van der Burg SH, Bijker MS, Welters MJ, Offringa R, Melief CJ. Improved peptide vaccine strategies, creating synthetic artificial infections to maximize immune efficacy. Adv Drug Deliv Rev. 2006;58:916-30.

12. Bijker MS, Melief CJ, Offringa R, van der Burg SH. Design and development of synthetic peptide vaccines: past, present and future. Expert Rev Vaccines. 2007;6:591-603.

13. Toes RE, Offringa R, Blom RJ, Melief CJ, Kast WM. Peptide vaccination can lead to enhanced tumor growth through specific T-cell tolerance induction. Proc Natl Acad Sci USA. 1996;93:7855-60.

14. Bijker MS, van den Eeden SJ, Franken KL, Melief CJ, Offringa R, van der Burg SH. CD8 + CTL priming by exact peptide epitopes in incomplete Freund's adjuvant induces a vanishing CTL response, whereas long peptides induce sustained CTL reactivity. J Immunol. 2007;179:5033-40.

15. Zwaveling S, Ferreira Mota SC, Nouta J, Johnson M, Lipford GB, Offringa $\mathrm{R}$, van der Burg SH, Melief CJ. Established human papillomavirus type 16-expressing tumors are effectively eradicated following vaccination with long peptides. J Immunol. 2002;169:350-8.

16. Bijker MS, van den Eeden SJ, Franken $\mathrm{KL}$, Melief CJ, van der Burg SH, Offringa R. Superior induction of anti-tumor CTL immunity by extended peptide vaccines involves prolonged, DC-focused antigen presentation. Eur J Immunol. 2008;38:1033-42.

17. Melief CJ, van der Burg SH. Immunotherapy of established (pre) malignant disease by synthetic long peptide vaccines. Nat Rev Cancer. 2008;8:351-60.

18. Gonda TA, Tu S, Wang TC. Chronic inflammation, the tumor microenvironment and carcinogenesis. Cell Cycle. 2009;8:2005-13.

19. Liu X, Ma X, Lei Z, Feng H, Wang S, Cen X, Gao S, Jiang Y, Jiang J, Chen $Q$, et al. Chronic inflammation-related HPV: a driving force speeds oropharyngeal carcinogenesis. PLoS One. 2015;10:e0133681.

20. Grivennikov SI, Greten FR, Karin M. Immunity, inflammation, and cancer. Cell. 2010;140:883-99.

21. Smyth MJ, Dunn GP, Schreiber RD. Cancer immunosurveillance and immunoediting: the roles of immunity in suppressing tumor development and shaping tumor immunogenicity. Adv Immunol. 2006;90:1-50.

22. Zhang YQ, Tsai YC, Monie A, Hung CF, Wu TC. Carrageenan as an adjuvant to enhance peptide-based vaccine potency. Vaccine. 2010;28:5212-9.

23. Kang TH, Mao CP, Lee SY, Chen A, Lee JH, Kim TW, Alvarez RD, Roden RB, Pardoll D, Hung CF, Wu TC. Chemotherapy acts as an adjuvant to convert the tumor microenvironment into a highly permissive state for vaccination-induced antitumor immunity. Cancer Res. 2013;73:2493-504.

24. Etchart N, Desmoulins PO, Chemin K, Maliszewski C, Dubois B, Wild F, Kaiserlian D. Dendritic cells recruitment and in vivo priming of CD8+CTL induced by a single topical or transepithelial immunization via the buccal mucosa with measles virus nucleoprotein. J Immunol. 2001;167:384-91.

25. Hakerud M, Selbo PK, Waeckerle-Men Y, Contassot E, Dziunycz P, Kundig TM, Hogset A, Johansen P. Photosensitisation facilitates cross-priming of adjuvant-free protein vaccines and stimulation of tumour-suppressing CD8 T cells. J Control Release. 2015;198:10-7.

26. Chesson CB, Huelsmann EJ, Lacek AT, Kohlhapp FJ, Webb MF, Nabatiyan A, Zloza A, Rudra JS. Antigenic peptide nanofibers elicit adjuvant-free CD8(+) T cell responses. Vaccine. 2014;32:1174-80.

27. Pepponi I, Stylianou E, van Dolleweerd C, Diogo GR, Paul MJ, Drake PM, Ma JK, Reljic R. Immune-complex mimics as a molecular platform for adjuvant-free vaccine delivery. PLoS One. 2013;8:e60855.

28. Faries MB, Hsueh EC, Ye X, Hoban M, Morton DL. Effect of granulocyte/ macrophage colony-stimulating factor on vaccination with an allogeneic whole-cell melanoma vaccine. Clin Cancer Res. 2009;15:7029-35. 
29. Slingluff CL Jr, Petroni GR, Olson WC, Smolkin ME, Ross MI, Haas NB, Grosh WW, Boisvert ME, Kirkwood JM, Chianese-Bullock KA. Effect of granulocyte/macrophage colony-stimulating factor on circulating CD8+ and CD4+ T-cell responses to a multipeptide melanoma vaccine: outcome of a multicenter randomized trial. Clin Cancer Res. 2009; 15:7036-44.

30. Salerno EP, Shea SM, Olson WC, Petroni GR, Smolkin ME, MCSkimming C, Chianese-Bullock KA, Slingluff CL Jr. Activation, dysfunction and retention of T cells in vaccine sites after injection of incomplete Freund's adjuvant, with or without peptide. Cancer Immunol Immunother. 2013;62:1149-59.

31. Hailemichael Y, Dai Z, Jaffarzad N, Ye Y, Medina MA, Huang XF, DortaEstremera SM, Greeley NR, Nitti G, Peng W, et al. Persistent antigen at vaccination sites induces tumor-specific CD8(+) T cell sequestration, dysfunction and deletion. Nat Med. 2013;19:465-72.

32. Shaw CA, Petrik MS. Aluminum hydroxide injections lead to motor deficits and motor neuron degeneration. J Inorg Biochem. 2009;103:1555-62.
33. Perricone C, Colafrancesco S, Mazor RD, Soriano A, Agmon-Levin N, Shoenfeld Y. Autoimmune/inflammatory syndrome induced by adjuvants (ASIA) 2013: unveiling the pathogenic, clinical and diagnostic aspects. J Autoimmun. 2013;47:1-16.

34. Peng S, Song L, Knoff J, Wang JW, Chang YN, Hannaman D, Wu TC, Alvarez $\mathrm{RD}$, Roden RB, Hung CF. Control of HPV-associated tumors by innovative therapeutic HPV DNA vaccine in the absence of CD4+ T cells. Cell Biosci. 2014:4:11.

35. Lin KY, Guarnieri FG, Staveley-O'Carroll KF, Levitsky HI, August JT, Pardoll DM, Wu TC. Treatment of established tumors with a novel vaccine that enhances major histocompatibility class II presentation of tumor antigen. Cancer Res. 1996;56:21-6.

36. Wu CY, Yang LH, Yang HY, Knoff J, Peng S, Lin YH, Wang C, Alvarez $\mathrm{RD}$, Pai SI, Roden RB, et al. Enhanced cancer radiotherapy through immunosuppressive stromal cell destruction in tumors. Clin Cancer Res. 2014;20:644-57.

\section{Submit your next manuscript to BioMed Central and we will help you at every step:}

- We accept pre-submission inquiries

- Our selector tool helps you to find the most relevant journal

- We provide round the clock customer support

- Convenient online submission

- Thorough peer review

- Inclusion in PubMed and all major indexing services

- Maximum visibility for your research

Submit your manuscript at www.biomedcentral.com/submit
() Biomed Central 